\title{
Association of Hypoglycaemia with Symptoms in the Newborn
}

\author{
A. D. GRIFFITHS \\ From the Department of Child Health, Welsh National School of Medicine, Cardiff
}

The symptoms produced by hypoglycaemia in the newborn include apnoeic attacks, alterations of muscle tone, 'juddery' movements of the limbs, convulsions, and reluctance to feed. These symptoms are not specific for hypoglycaemia, for they can be caused by a variety of conditions, such as perinatal anoxia, intracranial haemorrhage, meningitis, or hypocalcaemia.

When infants with both hypoglycaemia and symptoms have died, an acceptable cause for death other than the hypoglycaemia is often found at necropsy (Tynan and Haas, 1963; Chance and Bower, 1966). It is therefore possible that the presence of both hypoglycaemia and symptoms may in many cases also be merely coincidental.

The relation between hypoglycaemia, symptoms, and death has been investigated in 1000 consecutive admissions to the special care unit of a maternity hospital.

\section{Materials and Methods}

During the $3 \frac{1}{2}$ years from January 1964 to May 1967 1000 infants were admitted to the special care unit. The reason for admission was either because they weighed less than $2 \mathrm{~kg}$. at birth, were ill, or were at risk. 138 of these infants were born at home or in other hospitals, the remainder being among the 8736 live births which occurred in the Cardiff Maternity Hospital during this period.

All infants were fed within 6 hours of birth. The average daily calorie intake increased from approximately $17 \mathrm{cals} / \mathrm{kg}$. on the first day of life to approximately $90 \mathrm{cals} / \mathrm{kg}$. on the 7th day.

The infant was recorded as showing symptoms suggestive of hypoglycaemia if there were apnoeic attacks, irritability, 'juddery' movements, fits, or alterations of muscle tone.

Blood samples for glucose estimation were obtained by heel stab from all infants within 24 hours of admission to the unit, irrespective of the presence of symptoms. The postnatal age of infants at the time of initial sampling varied, but in the majority this was performed during the first day of life. Subsequent estimations were

Received June 27, 1968. usually performed if the initial glucose value was less than $20 \mathrm{mg} . / 100 \mathrm{ml}$. or if suggestive symptoms developed later. The heel was rubbed, but not otherwise warmed, and the interval between sampling and feeding varied. Blood glucose was estimated by a modification of the method of Watson (1962), and hypoglycaemia was diagnosed if the level was less than $20 \mathrm{mg} .100 \mathrm{ml}$.

Several conditions predispose to hypoglycaemia, and these underlying conditions rather than the hypoglycaemia itself may be responsible for symptoms. A further analysis was, therefore, undertaken of those infants with conditions known to predispose to hypoglycaemia, and the incidence of symptoms in hypoglycaemic and non-hypoglycaemic babies was compared within each group. The five groups were as follows: infants of diabetic mothers; infants with respiratory distress syndrome (RDS); anoxic infants (Apgar score 0-3); premature infants (less than 37 weeks' gestation); and infants of low birthweight for gestational age (more than 36 weeks but less than $2.5 \mathrm{~kg}$., 33-36 weeks but less than $1.5 \mathrm{~kg}$., 28-32 weeks but less than $1 \mathrm{~kg}$.). The relation of this classification to the intrauterine growth curves of Lubchenco et al. (1963) is shown in the Fig.

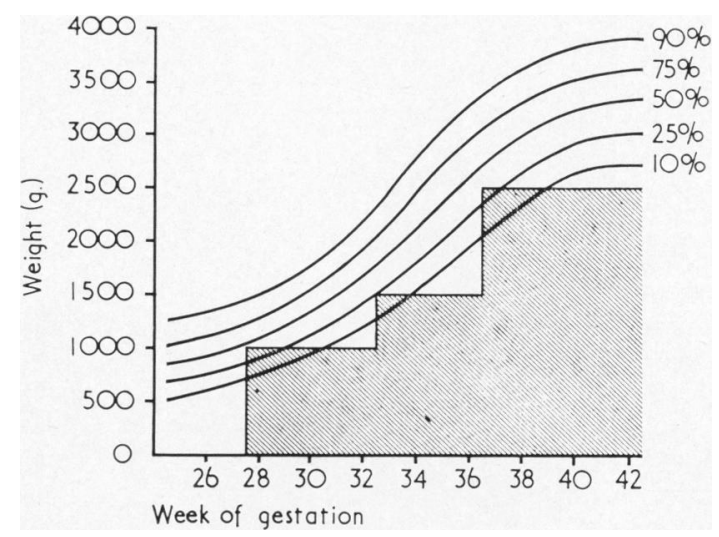

FIG.-Classification of low birthweight infants in relation to the intrauterine growth curves of Lubchenco et al. (1963). 
TABLE I

Comparison of Incidence of Symptoms in Hypoglycaemic and Non-hypoglycaemic Infants, Subdivided According to Factors which Predispose to Hypoglycaemia

\begin{tabular}{|c|c|c|c|c|c|c|c|c|}
\hline \multirow{2}{*}{$\begin{array}{l}\text { Predisposing } \\
\text { Factor }\end{array}$} & \multicolumn{3}{|c|}{ Hypoglycaemic Infants (148) } & \multicolumn{3}{|c|}{ Non-hypoglycaemic Infants (852) } & \multirow{2}{*}{$x^{2}$} & \multirow{2}{*}{$\mathbf{p}$} \\
\hline & Total & $\begin{array}{l}\text { No. With } \\
\text { Symptoms }\end{array}$ & $\begin{array}{c}\text { Incidence of } \\
\text { Symptoms (\%) }\end{array}$ & Total & $\begin{array}{l}\text { No. With } \\
\text { Symptoms }\end{array}$ & $\begin{array}{c}\text { Incidence of } \\
\text { Symptoms (\%) }\end{array}$ & & \\
\hline $\begin{array}{ll}\text { Anoxia } & \ldots \\
\text { Diabetic mother } & \ldots \\
\text { Prematurity } & \ldots \\
\text { RDS } & \text {.. } \\
\text { Low birthweight } \\
\text { for gestation age }\end{array}$ & $\begin{array}{r}52 \\
9 \\
74 \\
35 \\
44\end{array}$ & $\begin{array}{r}40 \\
6 \\
46 \\
24 \\
\\
24\end{array}$ & $\begin{array}{l}77 \\
67 \\
62 \\
69 \\
55\end{array}$ & $\begin{array}{r}264 \\
12 \\
344 \\
157 \\
155\end{array}$ & $\begin{array}{r}168 \\
9 \\
226 \\
112 \\
\\
80\end{array}$ & $\begin{array}{l}64 \\
75 \\
66 \\
71\end{array}$ & $\begin{array}{l}3 \cdot 409 \\
0 \cdot 176 \\
0 \cdot 335 \\
0 \cdot 106 \\
0 \cdot 118\end{array}$ & $\begin{array}{l}>0.05 \\
>0.05 \\
>0.05 \\
>0.05 \\
>0.05\end{array}$ \\
\hline
\end{tabular}

For significance at $p=0.05, \chi^{2}=3 \cdot 841$.

Some of the 1000 infants fell into none of these groups while others fell into, and are therefore included in, more than one group.

\section{Results}

Of the 1000 infants, 148 had one or more blood glucose levels of less than $20 \mathrm{mg}$. $/ 100 \mathrm{ml}$., and of these, 128 were born in the Cardiff Maternity Hospital. The incidence of hypoglycaemia was therefore $14.8 \%$ among admissions to the special care unit or $1.7 \%$ of total live births in the hospital. The age of onset of hypoglycaemia was accurately recorded in 114 of 148 infants, and was within the first 24 hours of life in $97(85 \%)$.

Among infants with proven hypoglycaemia $60 \cdot 1 \%$ were recorded as showing symptoms, compared with $52.9 \%$ in infants without hypoglycaemia. The difference in incidence is not significant $\left(\chi^{2}=2 \cdot 632 ; p=>0 \cdot 05\right)$.

Table I compares the incidence of symptoms in infants with and without hypoglycaemia when they are subdivided according to certain factors known to predispose to hypoglycaemia. In no instance was there a significant difference in the incidence of symptoms at the $p=0.05$ level, though this was almost attained in anoxic infants $\left(\chi^{2}=3 \cdot 409\right.$; $\mathrm{p}=\langle 0 \cdot 10:>0.05)$.

There were 22 deaths among the 148 infants with hypoglycaemia (14.9\%), and 124 deaths among the 852 infants without hypoglycaemia $(14 \cdot 6 \%)$. The death rates were thus the same in the two groups.

Necropsy records were available for 20 of the 22 infants with low blood glucose levels who died, and are summarized in Table II. Only in one infant (Case 4, Table II) could hypoglycaemia be regarded as the possible initiating factor in a chain of events ending in death. This infant had frequent cyanotic attacks which necessitated artificial ventilation, and though bronchopneumonia and a slight subarachnoid haemorrhage were found at necropsy, these could well have been secondary to artificial ventilation.

In all the remaining 19 infants there were gross pathological changes which appeared to be the cause of death.

\section{Discussion}

If hypoglycaemia is an important cause of symptoms in the newborn, as suggested by Brimblecombe (Brit. med. J., 1967), an increased incidence of symptoms would be expected in infants who are hypoglycaemic compared to those who are not. The present paper has shown that this expected increase does not occur, despite the fact that the method of selection of the infants studied favours such an association, for while all symptomatic infants would be admitted to the special care unit, asymptomatic infants with hypoglycaemia might be left undiagnosed in the main nurseries.

When infants of diabetic mothers, anoxic infants, prematures, infants with RDS, and infants of low birthweight for gestational age were studied separately, no statistical preponderanc $\epsilon$ of symptoms occurred in those with hypoglycaemia as compared to those without hypoglycaemia, though this was almost achieved in the anoxic group.

Thus, in the majority of infants, symptoms are seemingly unrelated to the presence of hypoglycaemia, and it follows that many infants do not develop symptoms despite glucose levels of less than $20 \mathrm{mg}$. $/ 100 \mathrm{ml}$.

This apparent resistance of the newborn to low glucose levels has been commented on by several workers (Hartmann and Jaudon, 1937; McKittrick, 1940; Norval, Kennedy, and Berkson, 1949; Haworth and Ford, 1960; Baird and Farquhar, 1962; Yu et al., 1965), and various explanations have been proposed. Stur (1964) suggested that the glucose values of blood obtained 
TABLE II

Clinical and Necropsy Findings in Hypoglycaemic Infants who Died

\begin{tabular}{|c|c|c|c|c|c|c|c|c|c|c|}
\hline \multirow{2}{*}{$\begin{array}{l}\text { Infant } \\
\text { No. }\end{array}$} & \multirow{2}{*}{$\begin{array}{c}\text { Matur- } \\
\text { ity (wk.) }\end{array}$} & \multirow{2}{*}{$\begin{array}{l}\text { Birth- } \\
\text { weight } \\
\text { (kg.) }\end{array}$} & \multirow{2}{*}{$\begin{array}{c}\text { Mater- } \\
\text { nal Age } \\
\text { (yr.) }\end{array}$} & \multirow{2}{*}{$\begin{array}{l}\text { Grav- } \\
\text { ida }\end{array}$} & \multirow{2}{*}{ Pregnancy } & \multicolumn{4}{|c|}{ Neonatal Course } & \multirow[b]{2}{*}{$\begin{array}{l}\text { Main Findings } \\
\text { at Necropsy }\end{array}$} \\
\hline & & & & & & $\begin{array}{c}\text { Glucose } \\
\text { Levels } \\
\text { (mg./100 ml.) }\end{array}$ & $\begin{array}{l}\text { Relevant } \\
\text { Therapy }\end{array}$ & Other Details & $\begin{array}{l}\text { Age at } \\
\text { Death }\end{array}$ & \\
\hline 1 & 37 & $1 \cdot 2$ & 35 & II & $\begin{array}{l}\text { Severe PET; } \\
\text { meconium- } \\
\text { stained } \\
\text { liquor }\end{array}$ & $\begin{array}{c}26 \mathrm{hr} .-9 \\
3 \mathrm{rd} \mathrm{dy} .-76\end{array}$ & $\begin{array}{l}\text { Oral } 10 \% \\
\text { fructose }+ \\
\text { milk only }\end{array}$ & $\begin{array}{l}\text { Apgar } 3 \text {; } \\
\text { raised res- } \\
\text { piratory rate } \\
\text { at } 38 \text { dy.- } \\
\text { 'cot' death }\end{array}$ & $\begin{array}{l}40 \\
\text { dy. }\end{array}$ & $\begin{array}{l}\text { Acute pulm. } \\
\text { infection; } \\
\text { cerebral } \\
\text { oedema with } \\
\text { uncal hernia- } \\
\text { tion }\end{array}$ \\
\hline 2 & 34 & $1 \cdot 6$ & 29 & III & $\begin{array}{c}\text { Severe APH; } \\
\text { Rh incom- } \\
\text { patibility }\end{array}$ & $\begin{array}{c}5 \mathrm{hr} .-355 \\
38 \mathrm{hr}-10 \\
51 \mathrm{hr}-79 \\
75 \mathrm{hr}--16\end{array}$ & $\begin{array}{l}\text { I.V. dextrose } \\
10 \% 4 \mathrm{ml} . / \\
\mathrm{hr} \text {. from } 3 \\
\mathrm{hr} \text {. for } \\
\text { RDS, dis- } \\
\text { continued } \\
\text { at } 80 \mathrm{hr} \text {. }\end{array}$ & $\begin{array}{l}\text { Apgar 1-2; } \\
\text { intubated; } \\
\text { RDS; } \\
\text { cyanotic } \\
\text { attacks; no } \\
\text { exchange } \\
\text { transfusions }\end{array}$ & $\begin{array}{l}4 \\
\text { dy. }\end{array}$ & $\begin{array}{l}\text { Massive pulm. } \\
\text { intraventricu- } \\
\text { lar, and sub- } \\
\text { dural haemor- } \\
\text { rhage, } \\
\text { resolving } \\
\text { hyaline mem- } \\
\text { brane disease; } \\
\text { haemolytic } \\
\text { disease }\end{array}$ \\
\hline 3 & 32 & NW & 20 & I & $\begin{array}{l}\text { Membranes } \\
\text { ruptured } 3 \\
\text { dy. pre- } \\
\text { maturely }\end{array}$ & $\begin{array}{l}2 \frac{1}{2} \mathrm{hr} .-18 \\
16 \mathrm{hr} .-4\end{array}$ & $\begin{array}{l}\text { I.V. dex- } \\
\text { trose } 10 \% \\
2 \mathrm{ml} . / \mathrm{hr} \text {. } \\
\text { from } 18 \mathrm{hr} . \\
\text { until death }\end{array}$ & $\begin{array}{l}\text { RDS; } \\
\text { cyanotic } \\
\text { attacks, } \\
\text { oedema }\end{array}$ & $\begin{array}{c}1 \\
\text { dy. }\end{array}$ & $\begin{array}{l}\text { Hyaline mem- } \\
\text { brane disease }\end{array}$ \\
\hline 4 & 33 & $2 \cdot 025$ & 18 & I & Hypertension & $\begin{array}{l}1 \mathrm{hr} .-12 \\
4 \mathrm{dy} \cdot-288 \\
5 \mathrm{dy} .-97\end{array}$ & $\begin{array}{l}\text { Chloral hyd- } \\
\text { rate, oral } \\
\text { fructose } \\
\text { + milk; } \\
\text { I.V. dex- } \\
\text { trose } 10 \% \\
5 \mathrm{ml} . / \mathrm{hr} \text {. } \\
\text { from } 3 \mathrm{rd} \\
\text { dy. until } \\
\text { death }\end{array}$ & $\begin{array}{l}\text { Rapid birth; } \\
\text { Apgar 9; } \\
\text { frequent } \\
\text { cyanotic } \\
\text { attacks; } \\
\text { IPPV }\end{array}$ & $\begin{array}{c}7 \\
\text { dy. }\end{array}$ & $\begin{array}{l}\text { Broncho- } \\
\text { pneumonia } \\
\text { patent ductus, } \\
\text { cerebral } \\
\text { oedema, slight } \\
\text { subarachnoid } \\
\text { haemorrhage }\end{array}$ \\
\hline 5 & 37 & $1 \cdot 9$ & 30 & VII & Uneventful & $2 \mathrm{hr} .-5$ & $\begin{array}{l}\text { I.V. dextrose } \\
5 \% \text { from } \\
5 \mathrm{hr} \text {. } \\
\text { infant trans- } \\
\text { ferred for } \\
\text { surgery; at } \\
\mathbf{4 8} \mathrm{hr} \text {. I.V. } \\
\text { therapy dis- } \\
\text { continued }\end{array}$ & $\begin{array}{l}\text { Imperforate } \\
\text { anus, low } \\
\text { thrombotest; } \\
\text { colostomy } \\
\text { within } 24 \\
\text { hr.; cardiac } \\
\text { arrest after } \\
\text { definitive } \\
\text { repair at } \\
10 \text { wk. }\end{array}$ & $\begin{array}{l}10 \\
\text { wk. }\end{array}$ & $\begin{array}{l}\text { Surgery for } \\
\text { imperforate } \\
\text { anus and } \\
\text { recto-vesical } \\
\text { fistula; single } \\
\text { umbilical } \\
\text { artery; absent } \\
\text { (R) kidney }\end{array}$ \\
\hline 6 & 35 & NW & 32 & III & APH at 35 wk. & 1 st dy. -6 & $\begin{array}{l}\text { [Records miss- } \\
\text { ing; other } \\
\text { details from } \\
\text { post-mortem } \\
\text { report] }\end{array}$ & $\begin{array}{l}\text { Melaena; } \\
\text { cold and } \\
\text { hypotonic; } \\
\text { abdominal } \\
\text { distension }\end{array}$ & $\begin{array}{c}3 \\
\text { dy. }\end{array}$ & $\begin{array}{l}\text { Haemorrhagic } \\
\text { disease, cere- } \\
\text { bral oedema, } \\
\text { and haemor- } \\
\text { rhage; } \\
\text { congenital } \\
\text { amputation } \\
\text { of }(R) \text { leg }\end{array}$ \\
\hline 7 & 30 & $1 \cdot 9$ & 42 & $\mathbf{V}$ & $\begin{array}{l}\text { Fetal distress; } \\
\text { meconium- } \\
\text { stained } \\
\text { liquor }\end{array}$ & $\begin{array}{c}4 \mathrm{hr} .-16 \\
29 \mathrm{hr} .-43 \\
58 \mathrm{hr} .-30 \\
90 \mathrm{hr} .-35\end{array}$ & None & $\begin{array}{l}\text { Dysmature, } \\
\text { low throm- } \\
\text { botest, } \\
\text { cerebral } \\
\text { signs, sep- } \\
\text { ticaemia }\end{array}$ & $\begin{array}{l}20 \\
\text { dy. }\end{array}$ & $\begin{array}{l}\text { Cerebral oedema } \\
\text { and anoxic } \\
\text { changes in } \\
\text { Ammon's } \\
\text { horn; monilia } \\
\text { septicaemia, } \\
\text { pneumonia, } \\
\text { agonal } \\
\text { intussception }\end{array}$ \\
\hline
\end{tabular}




\begin{tabular}{|c|c|c|c|c|c|c|c|c|c|c|}
\hline \multirow{2}{*}{$\begin{array}{c}\text { Infant } \\
\text { No. }\end{array}$} & \multirow{2}{*}{$\begin{array}{l}\text { Matur- } \\
\text { ity (wk.) }\end{array}$} & \multirow{2}{*}{$\begin{array}{l}\text { Birth- } \\
\text { weight } \\
\text { (kg.) }\end{array}$} & \multirow{2}{*}{$\begin{array}{c}\text { Mater- } \\
\text { nal Age } \\
(y r .)\end{array}$} & \multirow{2}{*}{$\begin{array}{l}\text { Grav- } \\
\text { ida }\end{array}$} & \multirow[b]{2}{*}{ Pregnancy } & \multicolumn{4}{|c|}{ Neonatal Course } & \multirow[b]{2}{*}{$\begin{array}{l}\text { Main Findings } \\
\text { at Necropsy }\end{array}$} \\
\hline & & & & & & $\begin{array}{c}\text { Glucose } \\
\text { Levels } \\
\text { (mg./100 ml.) }\end{array}$ & $\begin{array}{l}\text { Relevant } \\
\text { Therapy }\end{array}$ & Other Details & $\begin{array}{l}\text { Age at } \\
\text { Death }\end{array}$ & \\
\hline 8 & 36 & NW & 22 & I & Uneventful & $\begin{array}{c}11 \mathrm{hr} .-15 \\
18 \mathrm{hr} .-97 \\
2 \text { nd dy. }-92 \\
\text { 3rd dy. }-30 \\
11 \text { th dy. }-72\end{array}$ & $\begin{array}{l}\text { I.V. dextrose } \\
10 \% 5 \mathrm{ml} . / \\
\text { hr. from } 12 \\
\text { to } 48 \mathrm{hr} \text {. }\end{array}$ & $\begin{array}{l}\text { Breech deliv- } \\
\text { ery; peri- } \\
\text { natal anoxia, } \\
\text { hydro- } \\
\text { cephalus } \\
\text { after birth } \\
\text { trauma, } \\
\text { meningitis, } \\
\text { and sub- } \\
\text { dural } \\
\text { effusion }\end{array}$ & $\begin{array}{c}5 \\
\text { mth. }\end{array}$ & No necropsy \\
\hline 9 & 29 & NW & 19 & I & $\begin{array}{l}\text { APH; mem- } \\
\text { branes } \\
\text { ruptured for } \\
48 \mathrm{hr} .\end{array}$ & $\begin{array}{c}9 \mathrm{hr} .-42 \\
31 \mathrm{hr}-61 \\
55 \mathrm{hr} .-9 \\
61 \mathrm{hr} .-132 \\
4 \mathrm{dv} .-47 \\
5 \mathrm{dy} .-54\end{array}$ & $\begin{array}{l}\text { I.V. dextrose } \\
5 \% 2 \cdot 6 \\
\text { ml./hr. } \\
\text { from } 1 \mathrm{hr} \text {. } \\
\text { for } \mathrm{RDS} \text {; } \\
\text { stopped } \\
\text { spontaneous- } \\
\text { ly at } 42 \\
\text { hr. restarted } \\
\text { when hypo- } \\
\text { glycaemia } \\
\text { diagnosed } \\
\text { and con- } \\
\text { tinued until } \\
\text { death }\end{array}$ & $\begin{array}{l}\text { RDS; frequent } \\
\text { cyanotic } \\
\text { attacks } \\
\text { required } \\
\text { IPPV }\end{array}$ & $\begin{array}{r}5 \\
\text { dy. }\end{array}$ & $\begin{array}{l}\text { Broncho- } \\
\text { pneumonia; } \\
\text { (R) ventricular } \\
\text { haemorrhage; } \\
\text { cerebral } \\
\text { oedema }\end{array}$ \\
\hline 10 & 28 & NW & 28 & III & $\begin{array}{l}\text { APH; twin } \\
\text { pregnancy }(I)\end{array}$ & $\begin{array}{r}1 \frac{1}{2} \mathrm{hr} .-6 \\
7 \mathrm{hr} .-24 \\
20 \mathrm{hr} .-96\end{array}$ & \begin{tabular}{|l} 
I.V. dextrose \\
$5 \% 2.5 \mathrm{ml} . /$ \\
hr. from $4 \frac{1}{2}$ \\
hr. until \\
death; \\
chloral \\
hydrate
\end{tabular} & $\begin{array}{r}\text { Apgar 3; } \\
\text { RDS }\end{array}$ & $\begin{array}{l}1 \\
\text { dy. }\end{array}$ & $\begin{array}{l}\text { Hyaline mem- } \\
\text { brane disease, } \\
\text { intraventri- } \\
\text { cular, sub- } \\
\text { dural, and } \\
\text { subarachnoid } \\
\text { haemorrhage; } \\
\text { subcapsular } \\
\text { haematoma } \\
\text { of liver }\end{array}$ \\
\hline 11 & 28 & $1 \cdot 025$ & 28 & III & $\begin{array}{l}\text { APH; twin } \\
\text { pregnancy } \\
\text { (II) }\end{array}$ & $\begin{array}{r}1 \frac{1}{2} \mathrm{hr} .-9 \\
7 \mathrm{hr} .-10 \\
20 \mathrm{hr} .-30 \\
44 \mathrm{hr} .-45\end{array}$ & $\begin{array}{l}\text { I.V. dextrose } \\
5 \% 2 \cdot 5 \mathrm{ml} . / \\
\text { hr. from } 3 \frac{1}{2} \\
\text { hr. until } \\
\text { death }\end{array}$ & $\begin{array}{l}\text { Apgar 0-1; } \\
\text { intubated }\end{array}$ & $\begin{array}{l}3 \\
\text { dy. }\end{array}$ & $\begin{array}{l}\text { Intracranial } \\
\text { haemorrhage, } \\
\text { broncho- } \\
\text { pneumonia, } \\
\text { intussuscep- } \\
\text { tion, ruptured } \\
\text { gut, and } \\
\text { peritonitis }\end{array}$ \\
\hline 12 & 30 & $1 \cdot 7$ & 18 & I & $\begin{array}{l}\text { APH; mem- } \\
\text { branes } \\
\text { ruptured for } \\
3 \mathrm{dy} \text {. }\end{array}$ & $\begin{array}{r}8 \mathrm{hr} .-10 \\
32 \mathrm{hr} .-15 \\
56 \mathrm{hr} .-35 \\
80 \mathrm{hr} .-35\end{array}$ & $\begin{array}{l}\text { I.V. dextrose } \\
5 \% 5 \mathrm{ml} . / \\
\text { hr. from } 11 \\
\text { hr. to } 25 \mathrm{hr} \text {; } \\
\text { oral } 10 \% \\
\text { fructose } 4 \\
\text { ml. 3-hourly }\end{array}$ & $\begin{array}{l}\text { Assisted } \\
\text { breech } \\
\text { delivery; } \\
\text { rubella } \\
\text { syndrome; } \\
\text { exchange } \\
\text { transfusion } \\
\times 3 \text { for } \\
\text { haemolytic } \\
\text { anaemia }\end{array}$ & $\begin{array}{l}39 \\
\text { dy. }\end{array}$ & $\begin{array}{l}\text { Broncho- } \\
\text { pneumonia; } \\
\text { cerebral } \\
\text { oedema }\end{array}$ \\
\hline 13 & 36 & $1 \cdot 825$ & 25 & III & $\begin{array}{c}\text { APH; fetal } \\
\text { distress }\end{array}$ & $\begin{array}{r}3 \mathrm{hr} .-2 \\
8 \mathrm{hr} .-56 \\
26 \mathrm{hr} .-20 \\
50 \mathrm{hr} .-25\end{array}$ & $\begin{array}{l}\text { I.V. dextrose } \\
5 \% 2 \mathrm{ml} . / \\
\text { hr. from } 8 \frac{1}{2} \\
\text { hr. until } \\
\text { death; } \\
\text { hydrocorti- } \\
\text { sone } 20 \mathrm{mg} \text {. } \\
6-\text { hrly from } \\
10 \text { hr.; } \\
\text { chloral } \\
\text { hydrate }\end{array}$ & $\begin{array}{l}\text { Apgar 2; in- } \\
\text { tubated, } \\
\text { dysmature } \\
\text { with low } \\
\text { thrombo- } \\
\text { test, cere- } \\
\text { bral signs } \\
\text { and ECG } \\
\text { changes of } \\
\text { bundle- } \\
\text { branch } \\
\text { block }\end{array}$ & $\begin{array}{l}3 \\
\text { dy. }\end{array}$ & $\begin{array}{l}\text { Broncho- } \\
\text { pneumonia, } \\
\text { bilateral } \\
\text { haemorrhagic } \\
\text { papillary ne- } \\
\text { crosis }\end{array}$ \\
\hline
\end{tabular}




\begin{tabular}{|c|c|c|c|c|c|c|c|c|c|c|}
\hline \multirow{2}{*}{$\begin{array}{c}\text { Infant } \\
\text { No. }\end{array}$} & \multirow{2}{*}{$\begin{array}{l}\text { Matur- } \\
\text { ity (wk.) }\end{array}$} & \multirow{2}{*}{$\begin{array}{l}\text { Birth- } \\
\text { weight } \\
\text { (kg.) }\end{array}$} & \multirow{2}{*}{$\begin{array}{c}\text { Mater- } \\
\text { nal Age } \\
(y r .)\end{array}$} & \multirow{2}{*}{$\begin{array}{l}\text { Grav- } \\
\text { ida }\end{array}$} & \multirow[b]{2}{*}{ Pregnancy } & \multicolumn{4}{|c|}{ Neonatal Course } & \multirow{2}{*}{$\begin{array}{l}\text { Main Findings } \\
\text { at Necropsy }\end{array}$} \\
\hline & & & & & & $\begin{array}{c}\text { Glucose } \\
\text { Levels } \\
(\mathrm{mg} \cdot / 100 \mathrm{ml} .)\end{array}$ & $\begin{array}{l}\text { Relevant } \\
\text { Therapy }\end{array}$ & Other Details & $\begin{array}{l}\text { Age at } \\
\text { Death }\end{array}$ & \\
\hline 14 & 32 & $1 \cdot 76$ & 39 & IV & $\begin{array}{l}\text { APH due to } \\
\text { placenta } \\
\text { praevia }\end{array}$ & $\begin{array}{l}2 \frac{1}{2} \mathrm{hr} .-65 \\
24 \mathrm{hr} .-11 \\
48 \mathrm{hr} .-15\end{array}$ & $\begin{array}{l}\text { I.V. dextrose } \\
5 \% 1 \mathrm{ml} . / \\
\text { hr. from } 5 \frac{1}{2} \\
\text { hr. (for } \\
\text { RDS) until } \\
\text { death }\end{array}$ & $\begin{array}{l}\text { Caesarean sec- } \\
\text { tion; Apgar } \\
1-2 \text {; in- } \\
\text { tubated and } \\
\text { ventilated } \\
\text { by IPPV; } \\
\text { RDS; cere- } \\
\text { bral signs }\end{array}$ & $\begin{array}{l}3 \\
\text { dy. }\end{array}$ & $\begin{array}{l}\text { Intraventricular } \\
\text { haemorrhage; } \\
\text { basal cisterns } \\
\text { distended with } \\
\text { blood; hyaline } \\
\text { membrane } \\
\text { disease }\end{array}$ \\
\hline 15 & 39 & $1 \cdot 56$ & 27 & II & Uneventful & $\begin{array}{l}1 \frac{1}{2} \mathrm{hr} .-5 \\
10 \mathrm{hr} .-10 \\
21 \mathrm{hr} .-25 \\
45 \mathrm{hr} .-29 \\
69 \mathrm{hr} .-5\end{array}$ & $\begin{array}{l}\text { Chloral at } 1 \frac{1}{2} \\
\text { hr. oral } \\
\text { fructose } \\
10 \% \text { from } 5 \\
\text { hr. I. V. dex- } \\
\text { trose } 5 \% 3 \\
\text { ml. } / \text { hr. from } \\
24 \text { hr. re- } \\
\text { duced to } 1 \\
\text { ml./hr. at } 44 \\
\text { hr. and } \\
\text { continued } \\
\text { until death }\end{array}$ & $\begin{array}{l}\text { Severe peri- } \\
\text { natal } \\
\text { asphyxia, } \\
\text { Apgar 2-3; } \\
\text { intubated; } \\
\text { dysmature; } \\
\text { heart failure } \\
\text { with large } \\
\text { heart and } \\
\text { ischaemic } \\
\text { ECG } \\
\text { changes }\end{array}$ & $\begin{array}{l}3 \\
\text { dy. }\end{array}$ & $\begin{array}{l}\text { Haemorrhagic } \\
\text { broncho- } \\
\text { pneumonia; } \\
\text { congenital } \\
\text { heart disease } \\
\text { (VSD, PDA); } \\
\text { hydro-ureters }\end{array}$ \\
\hline 16 & 32 & $1 \cdot 775$ & 26 & IV & $\begin{array}{c}\text { Slight APH } \\
\text { at } 27 \text { wk. } \\
\text { after fall }\end{array}$ & $\begin{array}{r}1 \mathrm{hr} .-6 \\
19 \mathrm{hr} .-25\end{array}$ & $\begin{array}{l}\text { I. V. dextrose } \\
5 \% 5 \mathrm{ml} . \text { ! } \\
\text { hr. from } 1 \\
\text { hr. (to } \\
\text { administer } \\
\text { alkali for } \mathrm{pH} \\
6 \cdot 9 \text { ) and } \\
\text { continued } \\
\text { until death }\end{array}$ & $\begin{array}{l}\text { Perinatal as- } \\
\text { phyxia, } \\
\text { Apgar } \\
1-2 \text { intu- } \\
\text { bated; low } \\
\text { thrombotest; } \\
\text { ventilated } \\
\text { for broncho- } \\
\text { pneumonia }\end{array}$ & $\begin{array}{l}2 \\
\text { dy. }\end{array}$ & $\begin{array}{c}\text { Tentorial tear } \\
\text { with intra- } \\
\text { cranial hae- } \\
\text { morrhage; } \\
\text { broncho- } \\
\text { pneumonia }\end{array}$ \\
\hline 17 & 27 & NW & 31 & I & Uneventful & $\begin{array}{r}5 \mathrm{hr} .-19 \\
53 \mathrm{hr} .-10\end{array}$ & $\begin{array}{c}\text { Oral feeds of } \\
\frac{1}{2} \text {-strength } \\
\text { milk only }\end{array}$ & $\begin{array}{l}\text { Apgar 7-8; } \\
\text { extreme } \\
\text { prematur- } \\
\text { ity; apnoeic } \\
\text { attacks }\end{array}$ & $\begin{array}{l}2 \\
\text { dy. }\end{array}$ & $\begin{array}{l}\text { Almost complete } \\
\text { atelectasis }\end{array}$ \\
\hline 18 & 28 & 0.975 & 20 & III & APH & $\begin{array}{l}4 \frac{1}{2} \text { hr. }-10 \\
13 \text { hr. }-20 \\
34 \text { hr. }-35\end{array}$ & $\begin{array}{l}\text { Intragastric } \\
\text { drip of } \frac{1}{2}- \\
\text { strength } \\
\text { milk and } \\
\text { dextrose } 5 \%\end{array}$ & $\begin{array}{l}\text { Perinatal } \\
\text { asphyxia, } \\
\text { Apgar 1; } \\
\text { intubated; } \\
\text { RDS; venti- } \\
\text { lated for } \\
\text { cyanotic } \\
\text { attacks } \\
\text { from 5th } \\
\text { day until } \\
\text { death }\end{array}$ & $\begin{array}{c}7 \\
\text { dy. }\end{array}$ & No necropsy \\
\hline 19 & 27 & $1 \cdot 2$ & 24 & III & $\begin{array}{l}\text { No antenatal } \\
\text { care }\end{array}$ & $\begin{array}{c}6 \mathrm{hr} .-11 \\
10 \frac{1}{2} \mathrm{hr} .-10 \\
12 \mathrm{hr} .-12 \\
30 \mathrm{hr} .-30\end{array}$ & $\begin{array}{l}\text { Oral feeds of } \\
10 \% \text { fruc- } \\
\text { tose and } \\
\text { milk from } \\
12 \mathrm{hr} \text {. }\end{array}$ & $\begin{array}{l}\text { Apgar 3-4; } \\
\text { low throm- } \\
\text { botest; } \\
\text { apnoeic } \\
\text { attacks; } \\
\text { gastro- } \\
\text { enteritis }\end{array}$ & $\begin{array}{c}7 \\
\text { dy. }\end{array}$ & $\begin{array}{l}\text { Severe prematur- } \\
\text { ity, small } \\
\text { intracerebral } \\
\text { and subarach- } \\
\text { noid haemor- } \\
\text { rhage;Klebsiella } \\
\text { aerogenes } \\
\text { cultured } \\
\text { from intestine }\end{array}$ \\
\hline 20 & 28 & 0.7 & 25 & V & APH & $\begin{array}{r}15 \mathrm{hr} .-4 \\
21 \mathrm{hr} .-\quad 3 \\
25 \mathrm{hr} .-141 \\
39 \mathrm{hr} .-23 \\
44 \mathrm{hr} .-22 \\
63 \mathrm{hr} .-6 \\
87 \mathrm{hr}-{ }^{-} 13 \\
111 \mathrm{hr} .-17 \\
135 \mathrm{hr} .-3 \\
141 \mathrm{hr} .-10 \\
7 \mathrm{dy}-60\end{array}$ & $\begin{array}{l}\text { I.V. dextrose } \\
10 \% 1.5 \\
\text { ml./hr. } \\
\text { from } 23 \mathrm{hr} \text {; } \\
\text { changed to } \\
\text { dextrose } 5 \% \\
\text { at } 39 \mathrm{hr} \text {; } \\
\text { increased to } \\
3 \mathrm{ml} . / \mathrm{hr} \text {. at } \\
144 \mathrm{hr} \text { and } \\
\text { continued } \\
\text { until death; } \\
\text { hydro- } \\
\text { cortisone } \\
5 \mathrm{mg} . \mathrm{t} . \mathrm{d} . \mathrm{s} . \\
\text { from } 144 \mathrm{hr} .\end{array}$ & $\begin{array}{l}\text { Severe peri- } \\
\text { natal } \\
\text { asphyxia; } \\
\text { cyanotic } \\
\text { attacks }\end{array}$ & $\begin{array}{l}9 \\
\text { dy. }\end{array}$ & $\begin{array}{l}\text { Severe pre- } \\
\text { maturity, } \\
\text { intraventri- } \\
\text { cular and } \\
\text { subarachnoid } \\
\text { haemorrhage; } \\
\text { early broncho- } \\
\text { pneumonia; } \\
\text { thrombosis } \\
\text { of umbilical } \\
\text { vein }\end{array}$ \\
\hline
\end{tabular}


TABLE II-continued

\begin{tabular}{|c|c|c|c|c|c|c|c|c|c|c|}
\hline \multirow{2}{*}{$\begin{array}{c}\text { Infant } \\
\text { No. }\end{array}$} & \multirow{2}{*}{$\begin{array}{c}\text { Matur- } \\
\text { ity (wk.) }\end{array}$} & \multirow{2}{*}{$\begin{array}{l}\text { Birth- } \\
\text { weight } \\
\text { (kg.) }\end{array}$} & \multirow{2}{*}{$\begin{array}{l}\text { Mater- } \\
\text { nal Age } \\
\text { (yr.) }\end{array}$} & \multirow{2}{*}{$\begin{array}{c}\text { Grav- } \\
\text { ida }\end{array}$} & \multirow[b]{2}{*}{ Pregnancy } & \multicolumn{4}{|c|}{ Neonatal Course } & \multirow[b]{2}{*}{$\begin{array}{l}\text { Main Findings } \\
\text { at Necropsy }\end{array}$} \\
\hline & & & & & & $\begin{array}{c}\text { Glucose } \\
\text { Levels } \\
(\mathrm{mg} . / 100 \mathrm{ml} .)\end{array}$ & $\begin{array}{l}\text { Relevant } \\
\text { Therapy }\end{array}$ & Other Details & $\begin{array}{l}\text { Age at } \\
\text { Death }\end{array}$ & \\
\hline 21 & 33 & $1 \cdot 825$ & 36 & IV & Hypertension & $15 \mathrm{hr} \cdot-10$ & $\begin{array}{l}\text { I.V. dextrose } \\
5 \% 2 \mathrm{ml} . / \\
\text { hr. from } 3 \\
\text { hr. (for } \\
\text { RDS) } \\
\text { changed to } \\
\text { dextrose } \\
10 \% 3 \mathrm{ml} . / \\
\text { hr. when } \\
\text { hypogly- } \\
\text { caemia } \\
\text { diagnosed } \\
\text { and con- } \\
\text { tinued until } \\
\text { death }\end{array}$ & RDS & $\begin{array}{c}3 \\
\text { dy. }\end{array}$ & $\begin{array}{l}\text { Hyaline mem- } \\
\text { brane disease; } \\
\text { broncho- } \\
\text { pneumonia; } \\
\text { dilatation of } \\
\text { heart; hydrops } \\
\text { of meninges }\end{array}$ \\
\hline 22 & 34 & $1 \cdot 75$ & 39 & VIII & $\begin{array}{c}\text { Intrauterine } \\
\text { transfusion } \\
\text { for haemo- } \\
\text { lytic disease }\end{array}$ & $\begin{array}{l}24 \mathrm{hr} .-5 \\
28 \mathrm{hr} .-25 \\
30 \mathrm{hr} .-30 \\
48 \mathrm{hr}-15 \\
72 \mathrm{hr} .-20\end{array}$ & $\begin{array}{l}\text { I.V. dextrose } \\
5 \% 2 \mathrm{ml} . / \\
\text { hr. from } \\
\text { birth (for } \\
\text { RDS); } \\
\text { increased to } \\
\text { dextrose } \\
10 \% 3 \mathrm{ml} . \text { ! } \\
\text { hr. when } \\
\text { hypogly- } \\
\text { caemia } \\
\text { diagnosed; } \\
\text { reduced to } \\
\text { \% } 3 \mathrm{ml} . / \\
\text { hr. at } 35 \text { hr. } \\
\text { and con- } \\
\text { tinued until } \\
\text { death }\end{array}$ & $\begin{array}{l}\text { RDS; ex- } \\
\text { change } \\
\text { transfusion }\end{array}$ & $\begin{array}{c}4 \\
\text { dy. }\end{array}$ & $\begin{array}{l}\text { RDS; haemo- } \\
\text { lytic disease; } \\
\text { intraventri- } \\
\text { cular, intra- } \\
\text { cerebral, and } \\
\text { subarachnoid } \\
\text { haemorrhage } \\
\text { with cerebral } \\
\text { softening; } \\
\text { needle track } \\
\text { through liver }\end{array}$ \\
\hline
\end{tabular}

APH, antepartum haemorrhage; IPPV, intermittent positive pressure ventilation; NW, not weighed; PDA, patent ductus arteriosus; PET, pre-eclamptic toxaemia; RDS, respiratory distress syndrome; VSD, ventricular septal defect.

by heel stab were falsely low due to peripheral stasis, but Cornblath, Levin, and Gordon (1956) have shown that heel-prick blood has a 'true' sugar content similar to that of femoral artery blood. The findings of Edwards (1964) in calves and of Scopes (1964) in babies suggest a resistance of the brain of the neonate to hypoglycaemia, dependent upon its ability to metabolize lactate, symptoms being absent as long as the lactate level is adequate. It is, therefore, probable that the infant's resistance is in some way dependant on the biochemical and metabolic environment, which is rapidly changing during the neonatal period.

While the present statistical analysis shows that in the majority of infants symtoms are unrelated to hypoglycaemia, in one case (Case 4, Table II), it is possible that the hypoglycaemia was the underlying cause of death. Shelley and Neligan (1966) demonstrated that in a small proportion of cases hypoglycaemia was the cause of symptoms, and emphasized the importance of the diagnostic therapeutic test with a large dose of glucose (1-3 g.) intravenously. The incidence of such 'true' symptomatic hypoglycaemia is low, 2 per 1000 total births (Neligan, Robson, and Watson, 1963), 2.9 per 1000 total births (Cornblath, 1967), and 1.3 per 1000 term births (Zetterström, 1963), compared to the over-all incidence of infants with both hypoglycaemia and symptoms of $10 \cdot 1$ per 1000 in the present series, and a total incidence of hypoglycaemia (with or without symptoms) of 17 per 1000 live births.

Anderson, Milner, and Strich (1966), on the basis of 2 cases, imply that the finding of hypoglycaemia in the neonate is of serious import and that urgent therapy is required. The results of the present investigation serve to place these findings in perspective. While a diagnostic therapeutic test is indicated, especially if anoxia has occurred, the treatment of every infant with a blood glucose of less than $20 \mathrm{mg} . / 100 \mathrm{ml}$. seems unnecessary, and if the umbilical vein is used, therapy 
may be harmful (Scott, 1965; Sanerkin, Edwards, and Jacobs, 1966).

\section{Summary}

Studies were made of 1000 newborn infants admitted to a special care nursery. Hypoglycaemia (blood glucose level below $20 \mathrm{mg}$. $/ 100 \mathrm{ml}$.) was frequently found when looked for routinely $(17 / 1000$ live births). When infants with and without hypoglycaemia were compared, the incidence of symptoms did not differ significantly between the groups, and the death rates were identical.

In those hypoglycaemic infants who died, an acceptable cause of death other than the hypoglycaemia was established, with but one possible exception.

I am indebted to Professor A. G. Watkins and Dr. O. P. Gray for advice and permission to study the records of infants under their care, and to Miss Anne Mayled for secretarial assistance.

\section{REFERENCES}

Anderson, J. M., Milner, R. D. G., and Strich, S. J. (1966). Pathological changes in the nervous system in severe neonatal hypoglycaemia. Lancet, $2,372$.

Baird, J. D., and Farquhar, J. W. (1962). Insulin-secreting capacity in newborn infants of normal and diabetic women. ibid., 1,71 .

Brit. med. f. (1967). Babies at risk. In Medicine Today. 2, 559.

Chance, G. W., and Bower, B. D. (1966). Hypoglycaemia and temporary hyperglycaemia in infants of low birthweight for maturity. Arch. Dis. Childh., 41, 279.
Cornblath, M. (1967). In discussion to Cornblath, M., Segal, S., and Smith, C. A. Carbohydrate and energy metabolism in the newborn: an international exploration. Pediatrics, 39, 582 .

_, Levin, E. Y., and Gordon, H. H. (1956). Studies of carbohydrate metabolism in the newborn. 1. Capillary-venous differences in blood sugar in normal newborn infants. ibid., $18,167$.

Edwards, A. V. (1964). Resistance to hypoglycaemia in the newborn calf. f. Physiol. (Lond.), 171, $46 \mathrm{P}$.

Hartmann, A. F., and Jaudon, J. C. (1937). Hypoglycemia. f. Pediat., 11, 1 .

Haworth, J. C., and Ford, J. D. (1960). Blood-sugar in infants after lactose feeds. Lancet, $2,794$.

Lubchenco, L. O., Hansman, C., Dressler, M., and Boyd, E. (1963). Intrauterine growth as estimated from liveborn birthweight data at 24 to 42 weeks of gestation. Pediatrics, 32, 793.

McKittrick, J. B. (1940). Serial blood sugar determinations in normal newborn infants. F. Pediat., 16, 151.

Neligan, G. A., Robson, E., and Watson, J. (1963). Hypoglycaemia in the newborn. A sequel of intrauterine malnutrition. Lancet, 1, 1282.

Norval, M. A., Kennedy, R. L. J., and Berkson, J. (1949). Blood sugar in newborn infants. $\mathcal{F}$. Pediat, 34, 342.

Sanerkin, N. G., Edwards, P., and Jacobs, J. (1966). Pulmonary thrombo-embolic phenomena in the newborn. $\mathcal{F}$. Path. Bact., 91, 569.

Scopes, J. W. (1964). Hypoglycaemia in childhood (Discussion). Proc. roy. Soc. Med., 57, 1063.

Scott, J. M. (1965). Iatrogenic lesions in babies following umbilical vein catheterization. Arch. Dis. Childh., 40, 426.

Shelley, H. J., and Neligan, G. A. (1966). Neonatal hypoglycaemia. Brit. med. Bull., 22, 34.

Stur, O. (1964). Studies on the physiologic hypoglycemia of newborns. Biol. Neonat. (Basel), 6, 38.

Tynan, M. J., and Haas, L. (1963). Hypoglycaemia in the newborn. Lancet, $2,90$.

Watson, D. (1962). Enzymic determination of glucose and easily hydrolyzable glucose esters in blood. Analyt. Biochem., $3,131$.

Yu, J., Payne, W. W., Ifekwunigwe, A., and Stevens, J. (1965). Biochemical status of healthy premature infants in the first 48 hours of life. Arch. Dis. Childh., 40, 516.

Zetterström, R. (1963). Neonatal chemistry. Ann. N.Y. Acad. Sci., 111, 537 . 